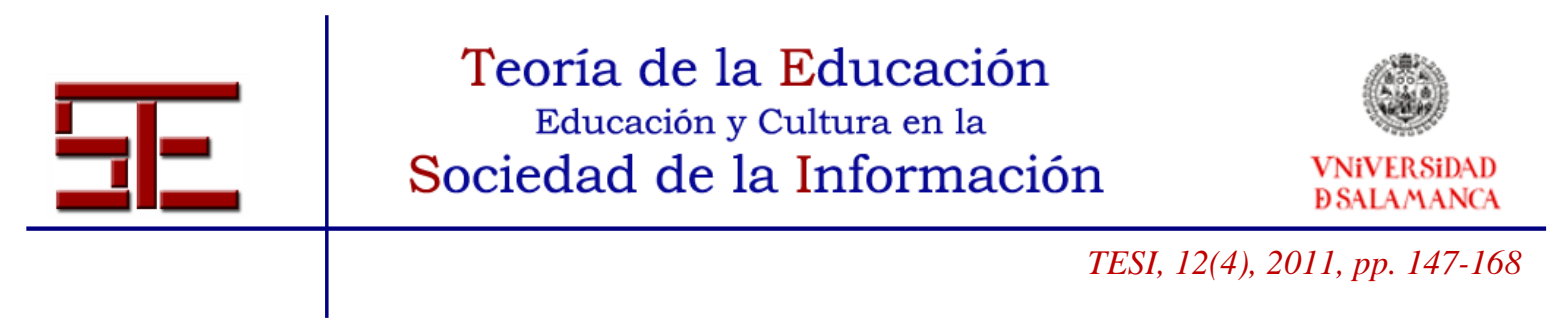

\title{
FORMACIÓN Y EDUCACIÓN CULTURAL EN LA SOCIEDAD DEL CONOCIMIENTO. ANÁLISIS EVOLUTIVO DE UNA ESTRATEGIA DIDÁCTICA COLABORATIVA EN EDUCACIÓN SUPERIOR
}

Resumen: El artículo explica una experiencia educativa desarrollada evolutivamente en la asignatura de Tecnología Educativa a través de la aplicación de un recurso de aprendizaje colaborativo, el Foro. Se realiza con estudiantes de tercer curso de Educador Social y Cultural en los Campus de Rímini y de Bolonia de la Facultad de Educación de la Universidad de Bolonia, a lo largo de dos cursos académicos consecutivos.

El proceso seguido orienta el trabajo en la asignatura de Tecnología Educativa no solo hacia una reflexión de naturaleza disciplinar, centrada en el uso de las TIC, sino también hacia un planteamiento de estrategias de aprendizaje colaborativo y de aprendizaje a lo largo de la vida, relacionadas con la adquisición de otras significativas competencias generales y transversales del Educador Social y Cultural involucradas en la adquisición de valores democráticos en una sociedad global.

A partir de la evaluación y el análisis del proceso educativo y de la experiencia de formación social en donde se desarrolla el Foro, las autoras hacen una propuesta de diseño didáctico desde el paradigma crítico y reflexivo, tras valorar diferentes observaciones sobre los resultados, relacionadas con los aspectos positivos y los límites de utilización del instrumento en el contexto universitario.

Palabras clave: TIC; Aprendizaje colaborativo; Educación Social y Cultural; Didáctica; Social Networking; Aprendizaje a lo largo de la vida; Valores democráticos.

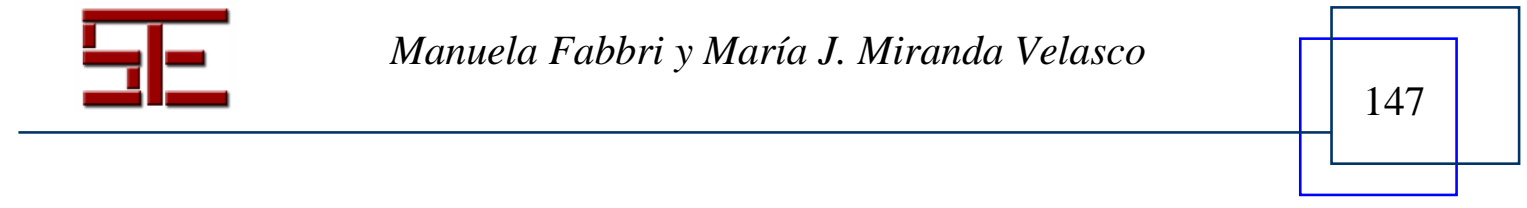




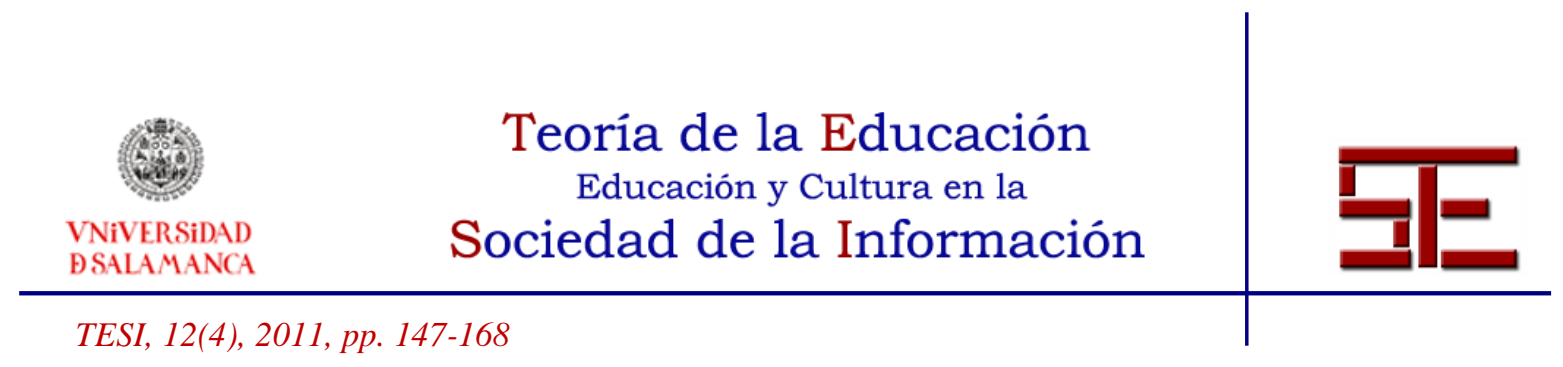

\title{
TEACHING AND CULTURAL EDUCATION IN THE KNOWLEDGE SOCIETY. EVOLUTIVE ANALYSIS OF A STRATEGY OF COLLABORATIVE LEARNING IN HIGHER EDUCATION
}

\begin{abstract}
This paper discuss in a pedagogical level an experience on the use of Forum as a telematic device. The part of e-learning comprised, together with contents and different kind of exercises, a forum. It was prepared for undergraduated students (third year) in Social and Cultural Education (Faculty of Education, University of Bologna). From a brief analysis of the context for the Forum on collaborative learning, authors present a description of the quantitative data from experience, some reflections about the research for techno-social goals, and extract some conclusions from positive elements and limits when using TICs in Higher Education system. From assessment and analysis of the educational process and experience of social formation that develops in the Forum, the authors present an instructional design proposal from the critical and reflective paradigm, after evaluating various comments on the results, related with the strengths and limitations of the instrument in the university context.

The conclusions guide the work in the subject of educational technology to not only a reflection of the disciplinary nature focused on the use of ICT, but also an approach to collaborative learning strategies and throughout lifelong learning related with the acquisition of general skills and other significant cross-Social and Cultural Educator, regarding the necessary democratic values in a global society.
\end{abstract}

Keywords: TIC; Collaborative Learning; Education Social and Cultural; Didactic; Social Networking; lifelong learning; Democratic values.

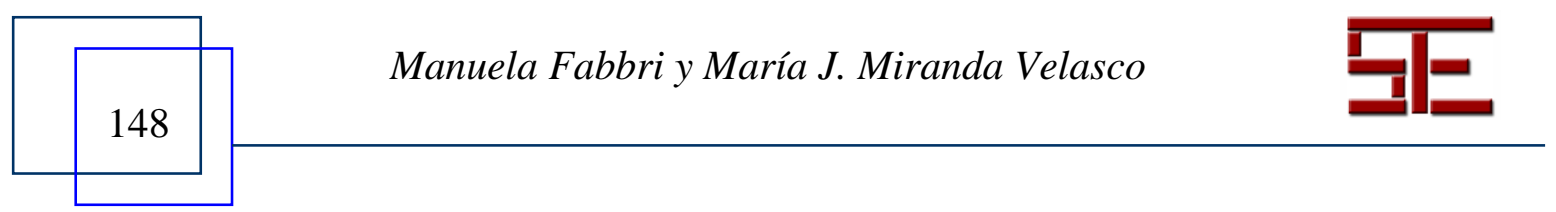




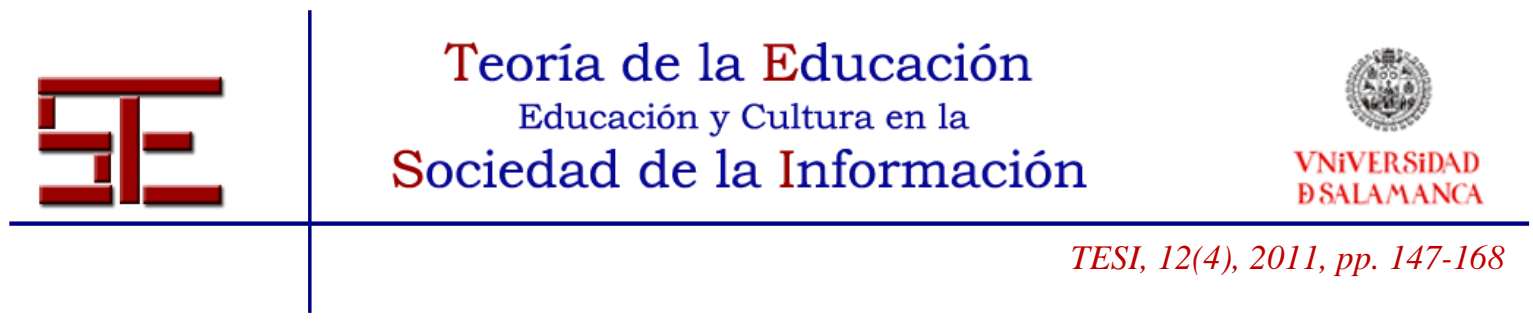

\title{
FORMACIÓN Y EDUCACIÓN CULTURAL EN LA SOCIEDAD DEL CONOCIMIENTO. ANÁLISIS EVOLUTIVO DE UNA ESTRATEGIA DIDÁCTICA COLABORATIVA EN EDUCACION SUPERIOR
}

Fecha de recepción: 31/10/2011; fecha de aceptación: 01/12/2011; fecha de publicación: 20/12/2011

\author{
Manuela Fabbri \\ m.fabbri@unibo.it \\ Universitá di Bologna (Italia) \\ María J. Miranda \\ mirandav@unex.es \\ Universidad de Extremadura
}

\section{INTRODUCCIÓN}

El artículo explica una experiencia educativa realizada en la Facultad de Educación, en los Campus de Rímini y de Bolonia, de la Universidad de Bolonia, con estudiantes de tercer curso de Educación Social y Cultural ${ }^{1}$ a lo largo de dos cursos académicos consecutivos utilizando un recurso didáctico de aprendizaje colaborativo, el Foro, en la asignatura de Tecnología Educativa.

Este estudio se inicia en el marco de una colaboración de internacionalización académica en Educación Superior, desde una perspectiva constructivista del uso de la tecnología educativa en el EEES (Miranda, 2010) llevada a cabo por las coautoras del trabajo en el curso académico 2008/2009 (Fabbri, 2009; Miranda, Fabbri, Guerra, 2010) que continúa en el curso académico 2009/2010 con la ampliación posterior de la investigación (Maeran, 2011).

Desde una concepción metodológica de investigación-acción en el proceso de enseñanza-aprendizaje (Lewin, 1973; Goyette y Lessard-Hérbert, 1988; Elliott, 1993; Colás Bravo, 1994) se analiza y se va construyendo evolutivamente el diseño metodológico que se empleará en la experiencia didáctica con los estudiantes. Todo ello en coherencia con la selección de las competencias implicadas en la formación del Educador Social y Cultural, relacionadas con la comunicación en red, incluidas en la dinámica de las comunidades de aprendizaje (Brown \& Campione, 1994). Se procede a plantear las guías para el diseño del Foro en función de los diferentes objetivos de

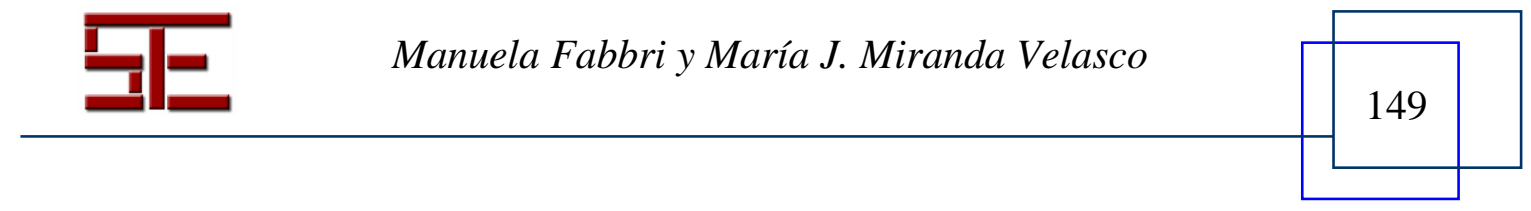






enseñanza de la asignatura. De manera concreta, se perfilan también los criterios didácticos para la construcción de una parrilla de decodificación que sirve para realizar el análisis de contenido de la producción de los estudiantes en el Foro.

En este artículo se presentan las reflexiones didácticas y una parte del análisis de la experiencia desarrollada evolutivamente relacionada con el desarrollo del trabajo, el análisis de las diferencias de los resultados obtenidos en los dos cursos académicos y la evolución metodológica en el segundo año, con una ampliación de los criterios elaborados en el primer curso.

Las conclusiones hacen referencia a las variables que pueden incidir en los métodos de enseñanza y procesos de aprendizaje colaborativo, y en la adquisición de competencias relacionadas con el pensamiento crítico de los futuros Educadores Sociales y Culturales.

\section{2.- PLANTAMIENTO TEÓRICO DEL TRABAJO}

La revolución digital que ha caracterizado las últimas décadas considera las TIC como el instrumento para desarrollar la sociedad de la información en lo que Marshall McLuhan (1988) denominó la Aldea Global para describir la interconexión humana a escala global, generada por los medios electrónicos de comunicación. La potencia de las nuevas tecnologías al poder llegar a millones de personas de todo el mundo es enorme. El trabajo de Castells (2009) plantea la encrucijada del siglo XXI en donde convergen el poder, la tecnología y la comunicación.

La introducción de las TIC en la sociedad contemporánea, especialmente en referencia a las dimensiones de la educación, es compleja, ya que presenta aspectos positivos para el aprendizaje y la socialización, al mismo tiempo que puede tener aspectos negativos (Guerra, 2010; Fabbri, Guerra 2010). Para estos autores que trabajan también desde la perspectiva de la Escuola Problematicista Bolognese 6 la acción de una Facultad de Educación debe de caracterizarse por la presencia de un proyecto educativo. Las nuevas tecnologías no sólo se utilizan por estar de moda o, simplemente por estar en el mercado de consumo de la cultura. La elección, según nuestro planteamiento, debe realizarse de manera que permita funcionalmente, por un lado, la participación de profesores y alumnos en la construcción del proyecto educativo y cultural de la Facultad $\mathrm{y}$, por otro, la apertura al contexto socioeducativo de la Comunidad a nivel local, nacional e internacional (Miranda, Fabbri, Guerra, 2010).

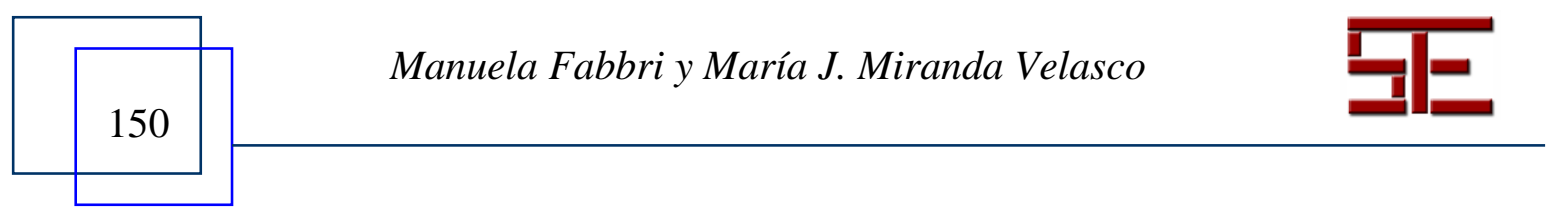




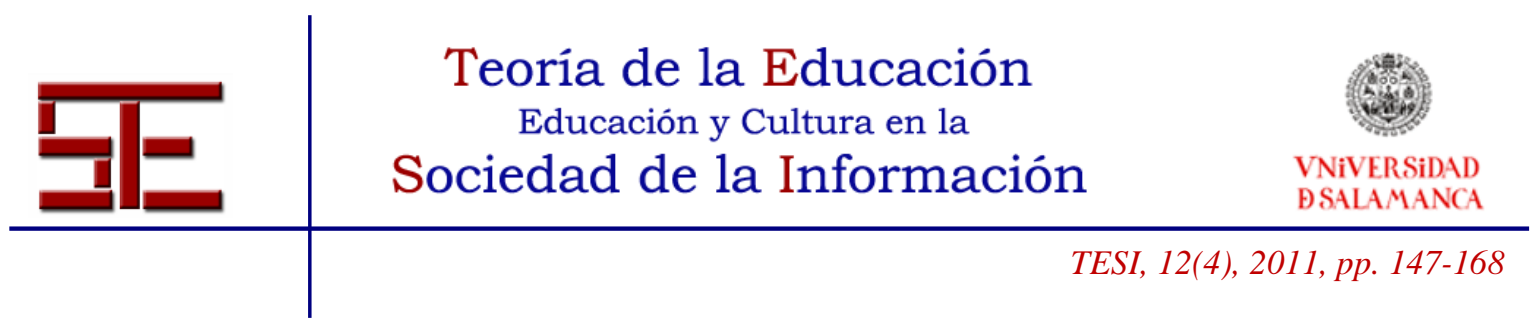

Diferentes planteamientos y estudios hacen hincapié en la permanencia de algunas dificultades para llevar a cabo el sentido profundamente revolucionario que supone la introducción de nuevas tecnologías digitales con respecto a las formas de construcción de la cultura, la mejora del desarrollo profesional, la realización de las intervenciones educativas en la escuela y actividades extracurriculares. Todavía se asiste en muchos casos a la aplicación meramente funcional de las TIC, poniendo en un plano menos importante de la práctica educativa las oportunidades que puede ofrecer la digitalización en la comunicación (Habermans, 1972), la toma de conciencia sociocultural, en el análisis de la realidad y en la trasformación de los contextos educativos, frente a la utilización de los métodos de enseñanza analógica (Ayuste y otros, 1994). Aún es necesaria la reflexión sobre la práctica y la formación y funciones de los tutores y profesionales de la educación para la correcta aplicación de las TIC a los entornos educativos (UNESCO, 1988; Cabero, 2005; Seoane, y otros, 2007; Valverde, 2010).

Desde esta perspectiva, consideramos que es necesario repensar e investigar sobre la práctica, porque el uso de tecnologías puede cambiar los procesos y resultados educativos aportando niveles de calidad mayores, establecer diferentes orientaciones en función de los objetivos de formación concretos en cada título y de las finalidades de formación integradora desde la perspectiva del aprendizaje a lo largo de la vida en el contexto universitario (Alberici, 2002; Costa, 2002, CEP, 2005).

Las Universidades actúan también como sistemas preventivos que pueden favorecer el óptimo desarrollo de programas relacionados con la implantación, difusión y aprovechamiento de los recursos tecnológicos. Todo ello implica la consideración de salvaguardar la dimensión educativa de las TIC y una reflexión didáctica de las acciones relacionadas con uso. En este contexto la finalidad será favorecer el empoderamiento de las personas para poder obtener el máximo beneficio de los recursos tecnológicos a favor del desarrollo social y no solo para ser instrumento de la reproducción social.

Este planteamiento se relaciona también con las teorías de Sociología de la Educación que consideran la necesidad de analizar la reproducción de los prejuicios y de las desigualdades sociales (Bernstein, 1990) que puede haber en los sistemas educativos para prevenirlos, desde una concepción de educación universitaria integral y consciente de su función en el desarrollo social.

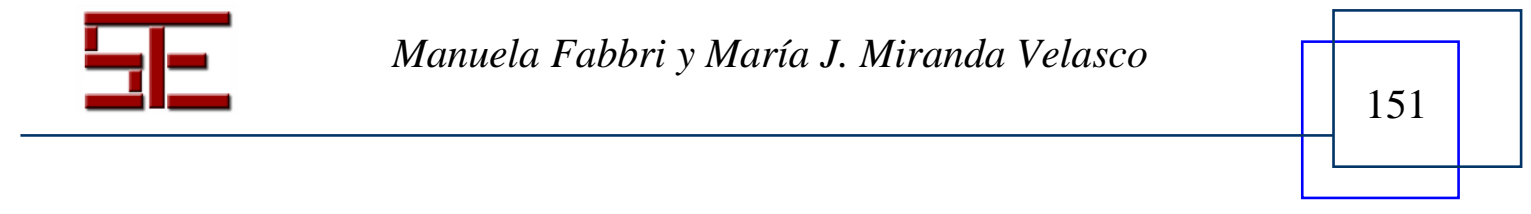






La finalidad relacionada con la formación de los futuros Educadores Sociales y Culturales consiste en destacar el valor del intercambio cultural y educativo, y en realizar un enfoque de los problemas socioeducativos, desde una perspectiva comprensiva, integradora y por competencias para el desarrollo personal y sociocultural (Delors, 1996), acorde a los objetivos europeos de democracia, inclusión y cohesión social (UNESCO, 2004).

El principal objetivo de la propuesta del Foro se orienta a ofrecer, por un lado, en coherencia con los objetivos de la asignatura, la oportunidad de experimentar una aplicación de social networking. Por otro, desarrollar las competencias del educador social 2.0 en la construcción del conocimiento, a través de una metodología colaborativa de discusión en red (OCDE, 2002; Granieri, 2006; UNESCO, 2008). Esto es, para potenciar la capacidad de comunicación, el análisis de la realidad social y el fomento de los valores de justicia social, equidad, solidaridad y ciudadanía. Todo ello a través de la aplicación de metodologías educativas activas, comprensivas, constructivistas y críticas (Nichols \& Allen-Brown, 1996; Calvani, 2005; Guerra, 2010; Miranda, 2010), que favorezcan la innovación en el EEES.

\section{3.- DISEÑO Y DESARROLLO DE LA ESTRATEGIA DIDÁCTICA COLABORATIVA}

\section{1.- Marco metodológico de la estrategia didáctica}

En el primer año la muestra se compone de 37 de los 50 estudiantes de Educación Social y Cultural que asisten regularmente a clase en el Campus de Rímini. En este caso el Foro ha acogido un total de 121 intervenciones. En el segundo año, la muestra está compuesta por 137 estudiantes matriculados en la misma asignatura de Tecnología Educativa del Campus de Bolonia. El total de las intervenciones es de 513.

La metodología empleada en el análisis de la producción de los estudiantes vertida al Foro y de las intervenciones efectuadas se realiza a través de procedimientos cualitativos que explicamos seguidamente. En primer lugar, se hace un vaciado del registro sistemático de las entradas al Foro de los estudiantes durante cinco semanas, en ambos cursos académicos. Posteriormente, se realiza una categorización del contenido vertido por los estudiantes al Foro y, finalmente, se aplica un tratamiento estadístico a las respuestas categorizadas. Los resultados de tipo cuantitativo se presentan en otro trabajo (Maeran, 2011).






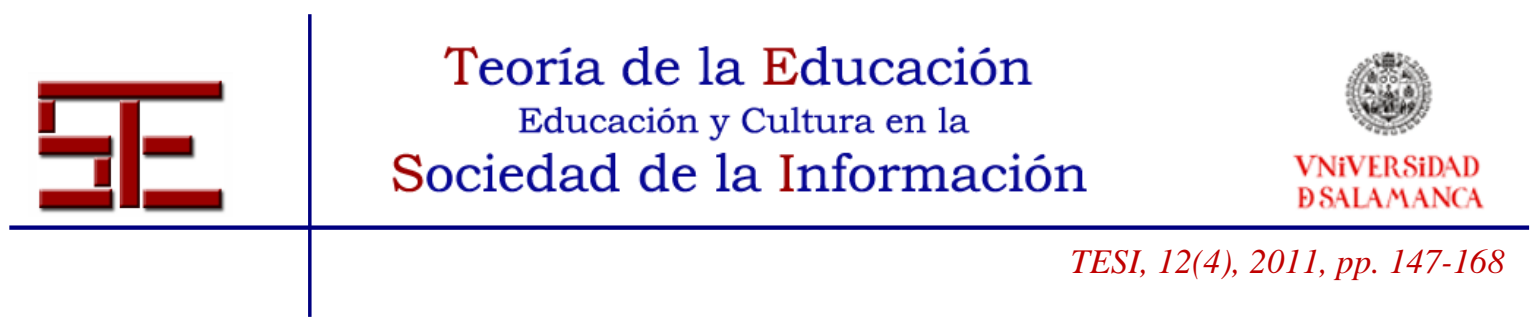

Al principio, el Foro se propone a los estudiantes como tarea formativa, pero no obligatoria. Sin embargo, en el segundo año la actividad tiene un carácter obligatorio. En ambos casos, la participación en el Foro proporciona una calificación sumativa.

El Foro se desarrolla a través de dos materiales multimedia seleccionados por su contenido, que reflejan la problemática intercultural que afecta a todas las esferas políticas y estatales que influyen en la cotidianidad de los ciudadanos. Se trata, concretamente, de dos fragmentos de 15 minutos cada uno, extraídos de las transmisiones de dos canales diferentes de la televisión italiana dedicados a los actuales problemas relacionados con la inmigración. Describen los enfrentamientos reales en territorio italiano de dos culturas diferentes. Las dos transmisiones son: una del programa L'infedele de Gad Lerner (www.gadlerner.it) del 21 de octubre del 2008, en el que participa el ministro Cota del Partido de La Lega Nord. El tema abierto trata sobre su propuesta de establecer una clase diferente para los estudiantes extranjeros (Classi Ponte). El segundo programa, Presa Diretta, de Ricardo Iacona (http://www.youtube.com/watch?v=GFGNzEpoQtw), emitido el 1 de febrero del 2009, plantea la difícil convivencia intercultural que existe en el barrio de la Pianura de Nápoles entre los autóctonos y los inmigrantes africanos. La crisis económica existente hace que las diferentes posturas se radicalicen. Esta situación contribuye a la creación de un debate en el que parecen prevalecer el racismo, el fundamentalismo y el rechazo del diálogo (Alessandrini, 2001).

\section{2.- Procedimiento seguido para la participación en el Foro.}

Los mencionados documentos audiovisuales se incluyen en la plataforma Moodle de la Universidad de Bolonia ${ }^{7}$, con la finalidad de contribuir a estimular el debate entre los estudiantes. Una vez vistos los materiales audiovisuales los estudiantes deben empezar la discusión en red relacionada estrechamente con los temas propuestos o abrir nuevos argumentos de discusión.

Los profesores, después de observar las primeras producciones de los estudiantes, reflexionan sobre el contenido y frecuencia de las intervenciones vertidas en la plataforma y les comunican, a través de un mensaje en el Foro, sus observaciones con una finalidad motivadora. Las observaciones del profesorado son en el siguiente sentido:

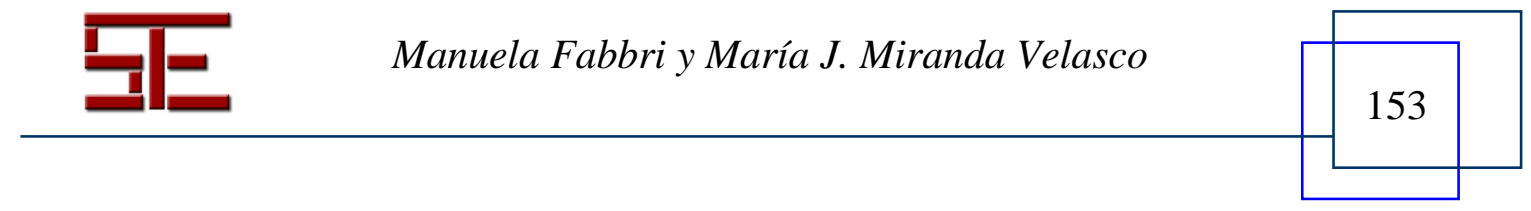




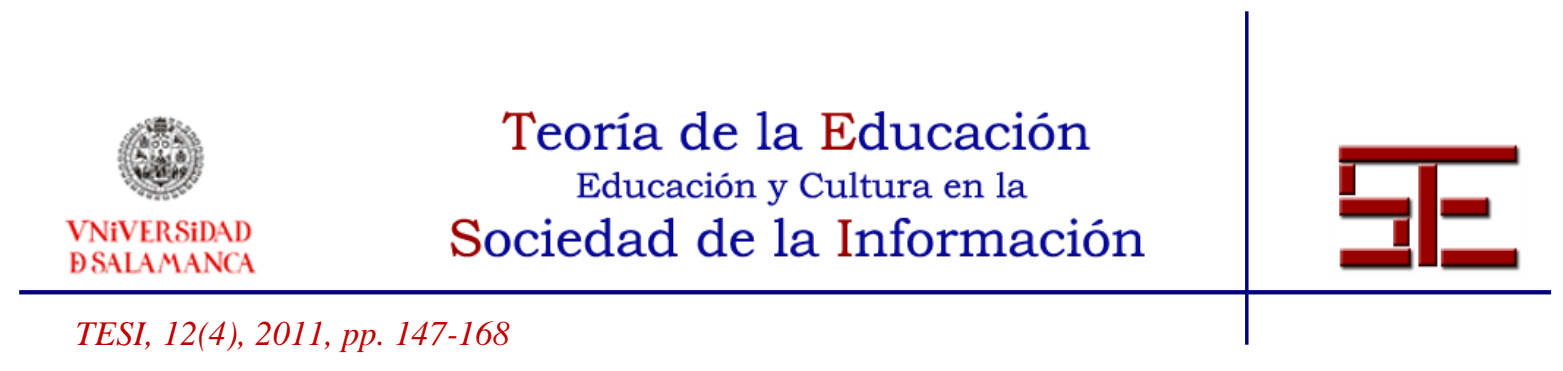

- Gran número de intervenciones, lo que demuestra una buena disposición a participar.

- Capacidad tanto para proponer nuevos temas para el debate, como la posibilidad de intervenir en temas ya propuestos por otros.

- Heterogeneidad de las intervenciones. Por un lado, composiciones basadas en conocimiento elaborado y, por otro lado, intervenciones basadas en posiciones más ideológicas que no demuestran un esfuerzo de investigación documental complementaria sobre los hechos.

- Presencia de referencias relacionadas con las competencias profesionales específicas y con los conocimientos adquiridos en diferentes disciplinas incluidas en los dos primeros años de la carrera.

Los análisis y reflexiones del proceso educativo seguido se hacen desde el principio, en diferentes momentos, no solo al final. De este modo se va obteniendo información que sirve para orientar la categorización de los contenidos.

3.3.- Construcción de la primera parrilla de análisis de las intervenciones de los estudiantes

Después de un primer análisis global de la marcha del Foro, en el primer año, se construye otra parrilla de análisis de las intervenciones de los estudiantes que describimos a continuación. Esta parilla esta subdividida por tipologías. Cada grupo contiene una serie de criterios que se utilizan para definir el tipo de intervenciones en el siguiente sentido:

a) Calidad de las informaciones.

Criterio 1. Informaciones precisas

Intervención dentro de la cual se introducen informaciones tomadas de fuentes citadas explícitamente, como artículos de periódicos, ensayos de naturaleza científica, fragmentos narrativos, textos de leyes, material iconográfico, etc.

\section{Criterio 2. Competencias profesionales}

Intervención dentro de la cual el estudiante se refiere de modo puntual a textos o palabras clave unidas a determinadas competencias de la función profesional del Educador Social y Cultural.

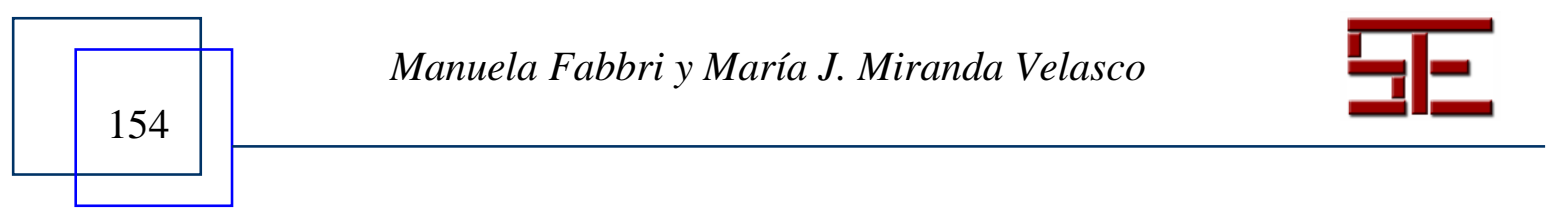




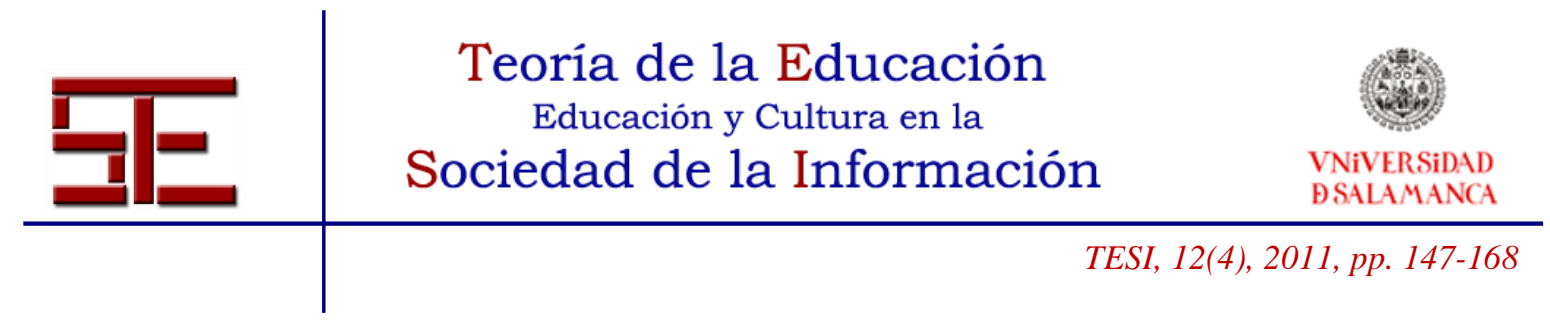

b) Clima político de la intervención

Criterio 3. Carácter "acogedor"

Intervención que representa una actitud mental de naturaleza positiva, de acogida, en los procesos de integración del emigrante, sea esto defendido o no con argumentos adecuados.

Criterio 4. Carácter "defensivo"

Intervención que representa una actitud mental de naturaleza defensiva o, en cualquier caso, diferente y reactiva sobre los procesos de integración del emigrante, sea esto defendido o no con argumentos adecuados.

c) Perfil de la participación

Criterio 5. Apertura de un nuevo tema

Intervención que explota las posibilidades ofertadas por la plataforma Moodle de abrir nuevos temas dentro del Foro relacionados con los contenidos propuestos.

Criterio 6. Respuesta puntual a un tema

Intervención que se inserta dentro de un tema ya presente en la plataforma.

Criterio 7. Retoma el lanzamiento de los dos materiales audioviuales Intervención que utiliza de manera relacionada los dos materiales multimedia propuestos inicialmente como provocación de apertura del Foro.

3.4.- Construcción de la segunda parrilla de análisis de las intervenciones de los estudiantes

En el segundo año se plantea una ampliación de los 7 criterios de análisis iniciales ${ }^{8}$, en el siguiente sentido:

a) Perfil de la participación

Foro

Criterio 1. Presentación de un tema uniforme coherente con el propuesto en el

Acción que abre un tema que, sin asumir los temas presentados en la propuesta de materiales multimedia para provocar la apertura del Foro, tiene características de

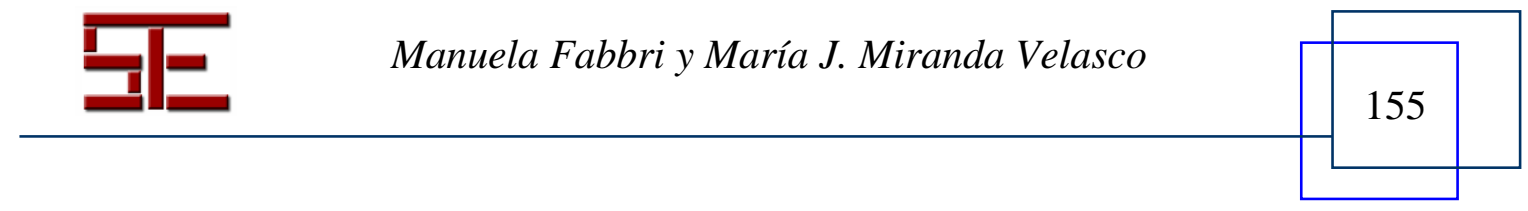




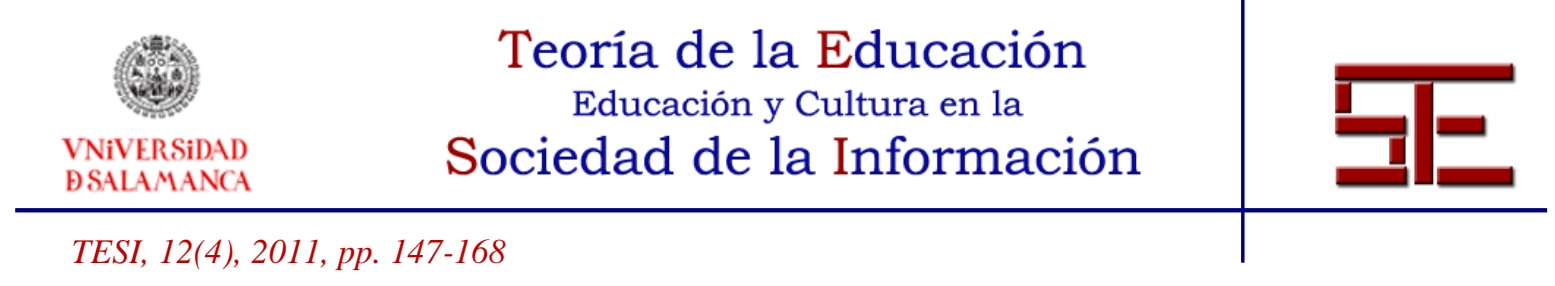

homogeneidad en cuanto al tema general de la interculturalidad y las actitudes hacia la diversidad.

Criterio 2. Presentación de un tema uniforme no coherente con el propuesto en el Foro

Acción que abre un tema que no tiene en cuenta el contenido de los dos materiales multimedia ofrecidos en el Foro

Criterio 3. Intervención de monitoreo

Intervención realizada por el mismo estudiante que inicia el debate y sigue su desarrollo.

\section{b) Calidad de la participación}

Criterio 4. Intervención de aprobación

Intervención que incorpora en el contenido las observaciones formuladas por los demás, dándoles crédito.

Criterio 5. Intervención discrepante

Intervención que expresa en el contenido los comentarios de desacuerdo con otros realizados por los compañeros y compañeras.

Criterio 6. Intervención que pone de relieve los problemas de interpretación de los mensajes de los demás

Intervención que responde a otra intervención en la que se demuestra que no han comprendido realmente el significado del contenido expresado.

Criterio 7. Intervención de respuesta a un discurso discrepante

Intervención que pretende dar respuesta a una intervención discrepante.

Criterio 8. Intervención neutral

Intervención que incorpora en el contenido las teorías de la interpretación expuesta por otros, pero sin expresar su opinión, sin decir si está de acuerdo o en desacuerdo.

c) Tipo de participación

Criterio 9. Declaración personal

En la que el alumno expresa su opinión personal o su interpretación, y fundamentación crítica de un determinado evento.

Criterio 10. Intervención de comentarios sobre el procedimiento metodológico Intervención que incorpora comentarios sobre la metodología seguida.

Criterio 11. Intervención resumen

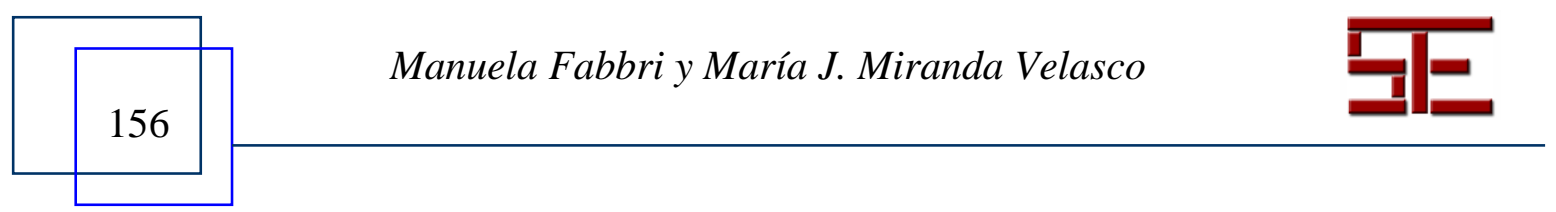




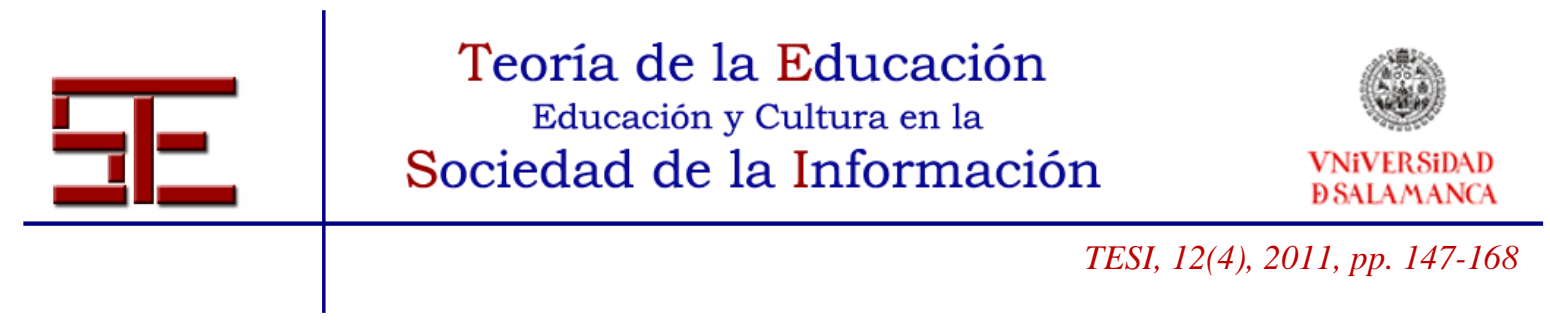

Intervención que manifiesta los diversos argumentos expresados anteriormente por otros compañeros.

Criterio 12. Intervención que abre nuevas cuestiones problemáticas Intervención que, a partir de ciertos temas previamente investigados, se abre a nuevas preguntas de investigación y discusión.

d) Solicitud de la colaboración/apoyo

Criterio 13. Intervención de petición de apoyo

Cuando un estudiante pide ayuda y/o aclaraciones a la comunidad, con el fin de entender más a fondo el tema en cuestión.

Criterio 14. Intervención que responde a una solicitud de intervención/apoyo Cuando un estudiante responde a una petición de ayuda y / o aclaración.

e) Calidad narrativa de la intervención

Criterio 15. Calidad en el lenguaje escrito

Acción que no presenta particulares errores gramaticales, ni sintácticos.

Criterio 16. Expresión escrita con elementos de la jerga digital

Intervención que contiene emoticonos u otros elementos típicos de expresión escrita en los jóvenes digitales.

Criterio 17. Expresión escrita incorrecta, gramaticalmente y sintaxis desarticulada Intervención que contiene evidentes errores gramaticales o de sintaxis.

Criterio 18. Intervención extensa

Intervención que supera las 15 líneas.

\section{4.- RESULTADOS DEL ANÁLISIS DE LA EXPERIENCIA EDUCATIVA EN RED}

En el primer y segundo año, las producciones se centran en la observación de tres temas diferentes relacionados con los presupuestos teóricos que fundamentan el trabajo, los cuales ya hemos hecho referencia en la primera parte. Esto es: Calidad de la información aportada en las intervenciones, competencias adquiridas en la formación universitaria y Aspectos sociopolíticos y valores democráticos en las Intervenciones, que, de manera resumida y analizando las diferencias evolutivas, presentamos a continuación.

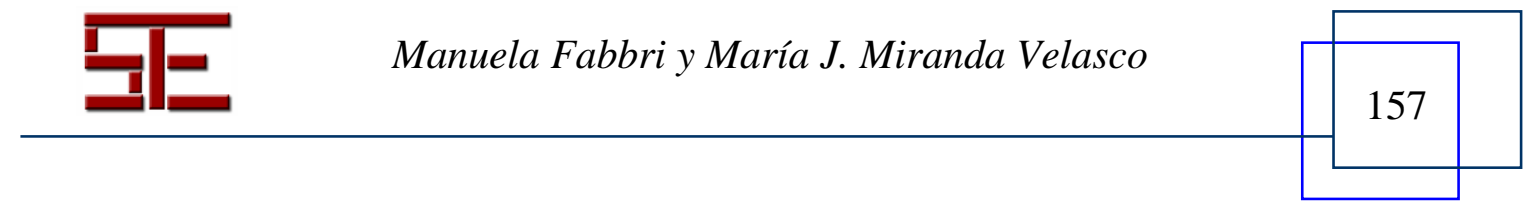




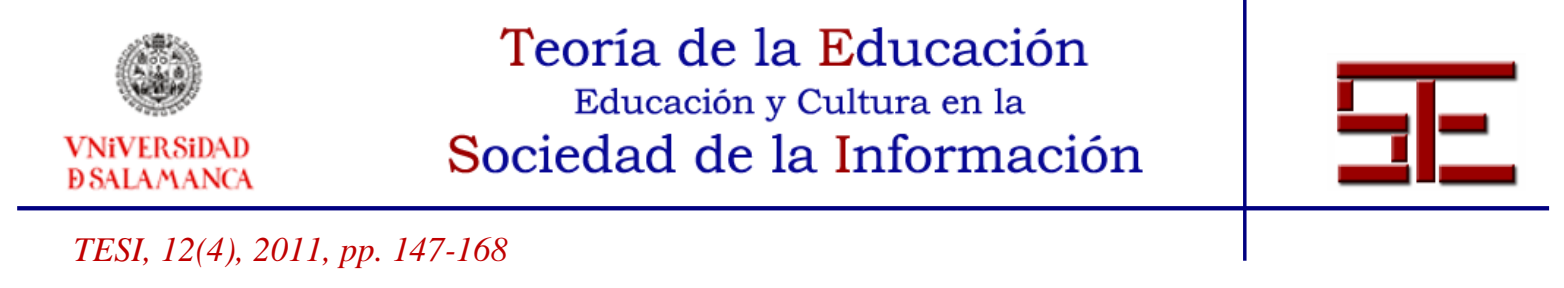

\subsection{Calidad de la información aportada en las intervenciones}

En el primero año, en el que las indicaciones del profesor estaban menos definidas, el porcentaje de las intervenciones que se refieren a información de tipo preciso es bastante escaso: $17 \%$. Mientras que en el segundo año el porcentaje aumenta al $38 \%$.

Las fuentes a las que los escritos de los estudiantes hacen referencia en sus argumentos son prevalentemente fuentes "calientes", es decir, son fragmentos tomados de Internet o artículos cotidianos, mientras que solo en un caso se hace referencia a un artículo de rigor como el de la Constitución.

Véase, por ejemplo, un fragmento de una intervención en el que el estudiante especifica la fuente utilizada.

\section{Re: Las mezquitas en Italia}

[...] La misma historia de Italia está diseminada de multi e interculturalidad, con tan sólo ir a Palermo te das cuenta: cultura árabe y normanda conviven en espléndidas arquitecturas, una mezcla que ha dado origen incluso a nuevos estilos artísticos, únicos en el mundo (http://en.wikipedia.org/wiki/Arab-Norman_culture: el link es ingles porque la versión italiana de Wikipedia no está traducida). [...]

En otros casos se hace referencia a textos de leyes o también a la misma Constitución italiana.

\section{Re: Emigrantes y derecho a los servicios}

Querría expresarme sobre la cuestión de la salud y por tanto sobre el disfrute de este servicio, recordando que existe una ley (Ley 40 de julio de 1998) que establece la igualdad absoluta entre ciudadanos italianos e inmigrantes regularizados en Italia. [...].

Solo una estudiante ha participado en el Foro utilizando códigos diferentes al escrito; lo ha hecho, además, aportando una imagen tomada de internet que contiene una inscripción que dice:Inmigrantes, por favor no nos dejéis solos con los italianos.

\subsection{Competencias adquiridas en la formación universitaria.}

Si tenemos en cuenta el criterio relativo a intervención que afecta a los contenidos que se relacionan con las competencias profesionales, en el primer y segundo año, el $25 \%$ de los estudiantes coinciden en el tipo de expresión.

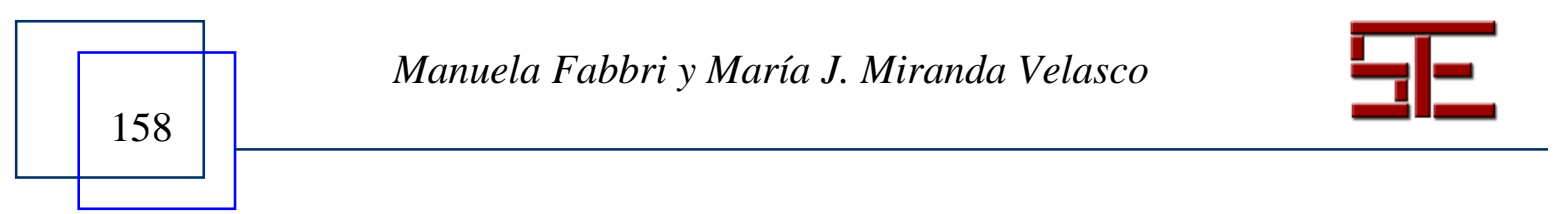




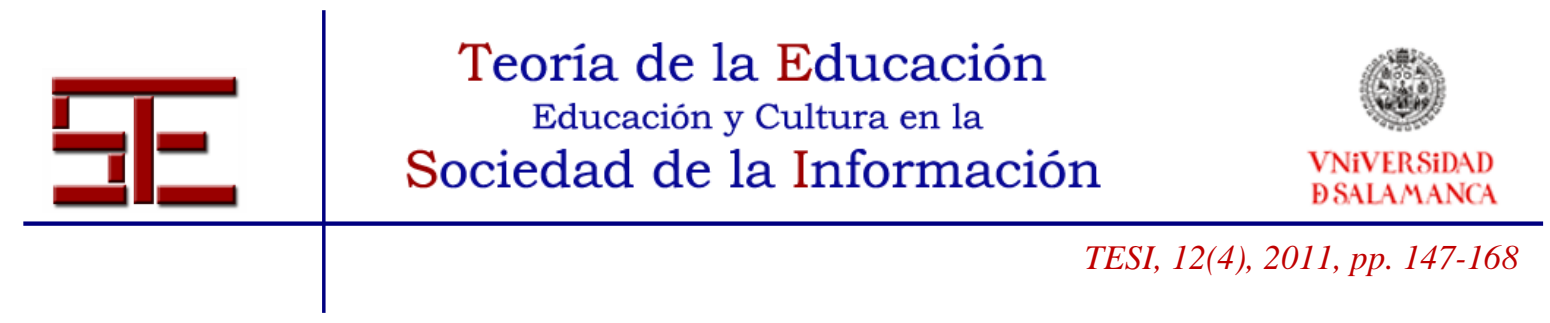

Vemos seguidamente un ejemplo de referencias más explícitas a algún rasgo de la cultura profesional:

\section{Re: Las mezquitas en Italia}

[...] Pensemos todos en modo particular, nosotros, Educadores Sociales en grado de poder hacer algo positivo que puede marcar un cambio... no es solo asistencia o sobrevivencia pero es sobre todo creer en un empowerment que conmueva al individuo y a la sociedad nacional e internacional [...].

Teniendo en cuenta que los estudiantes están en el último año de su formación, estos datos nos indican que hay una parte significativa de ellos que no son capaces de demostrar las competencias propuestas en el título de grado, haciendo intervenciones superficiales y sin fundamentación científica en sus argumentos profesionales.

En consecuencia, consideramos que es necesario desarrollar y fortalecer un modelo didáctico más constructivista, que incluya la didactica por competencias, desde la perspectiva crítica.

En este sentido la Tecnología Educativa puede ayudar a desarrollar recursos didácticos que potencien las competencias de los estudiantes en el marco de los objetivos de la innovación educativa (Calvani, Sorzio, Varisco, 1997; Guerra, 2010; Miranda, Guerra, Fabbri, Meneses, 2010).

\subsection{Aspectos sociopolíticos y valores democráticos en las intervenciones.}

La tercera área de significado analizada es la presencia de intervenciones con elementos referidos a una actitud hacia la integración acogedora o a una intervención sustancialmente defensiva en las expresiones escritas de los estudiantes.

En el primer año, las intervenciones de carácter acogedor representan un porcentaje notablemente mayor $(77 \%)$ respecto a las de carácter sustancialmente defensivo (23\%), mientras en el segundo año, las intervenciones de fondo acogedor representan un porcentaje notablemente mayor (96\%) respecto a las de carácter defensivo (4\%).

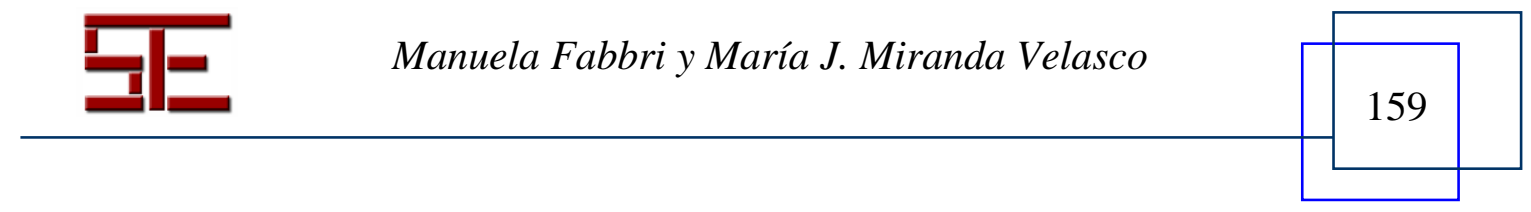




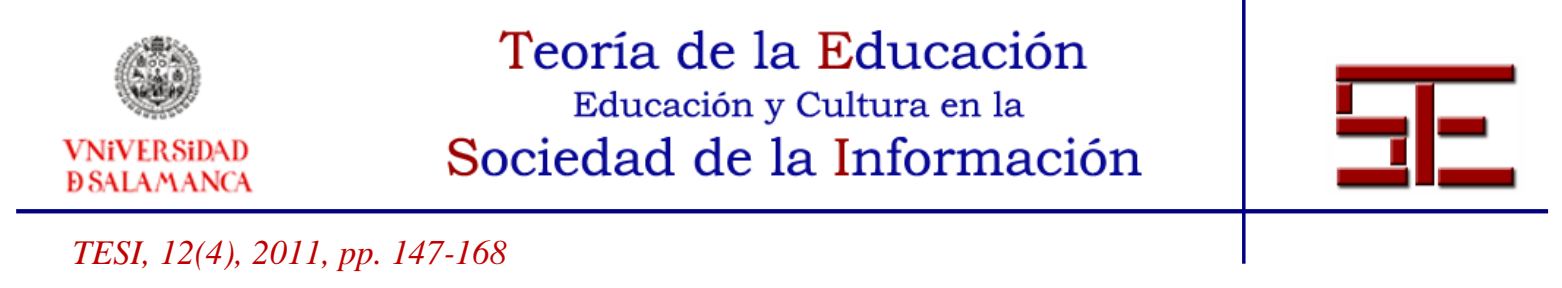

Considerando los datos del primer año, y que los participantes del Foro son estudiantes de Educación Social y Cultural, sorprende que en los resultados de los análisis de sus producciones haya un $23 \%$ de contenidos de tipo defensivo y no de aceptación del hecho multicultural en nuestra sociedad actual. Si las actitudes forman parte de sus competencias profesionales, los resultados obtenidos por un cuarto de la muestra no son aceptables en términos educativos.

Profundizando más en el análisis de contenido, se observa que en el caso de las intervenciones de carácter acogedor es mayor la presencia de información precisa (20\%), frente al $7 \%$ de la información precisa de los que han hecho intervenciones de tipo defensivo. Este resultado nos informa del carácter más emocional y espontáneo, que razonado y elaborado, en las intervenciones de los estudiantes.

Una relación similar se observa entre las competencias profesionales contenidas dentro de las intervenciones de los acogedores (31\%) y de los defensivos (7\%). Los datos relativos a la relación entre actitudes de integración y las competencias profesionales en los Educadores Sociales y Culturales se valoran más positivamente por su relación con las competencias democráticas relacionadas con la prevención de las desigualdades sociales, la justicia, equidad, explícitas en los programas formativos de la titulación. Concretamente, las competencias relacionadas con la educación en valores y la orientación de la profesión hacia la atención social y la integración.

Considerando los datos del segundo año, podemos decir que los datos reflejan que los estudiantes de Educación Social y Cultural han adquirido las actitudes acordes a lo esperado en su formación.

\subsection{Aspectos didácticos}

En coherencia con los objetivos didácticos de la experiencia, y con los resultados del análisis y evaluación de la práctica, planteamos al mismo tiempo la necesidad de considerar diferentes aspectos didácticos que pueden ser tenidos en cuenta en diferentes momentos y aspectos de la planificación del proceso de enseñanza-aprendizaje utilizando este recurso tecnológico. En este sentido, destacamos: la modalidad de apertura del Foro, la inclusión del Foro dentro del programa de la asignatura, la calidad de la comunicación en el Foro y la potenciación del rol del estudiante.

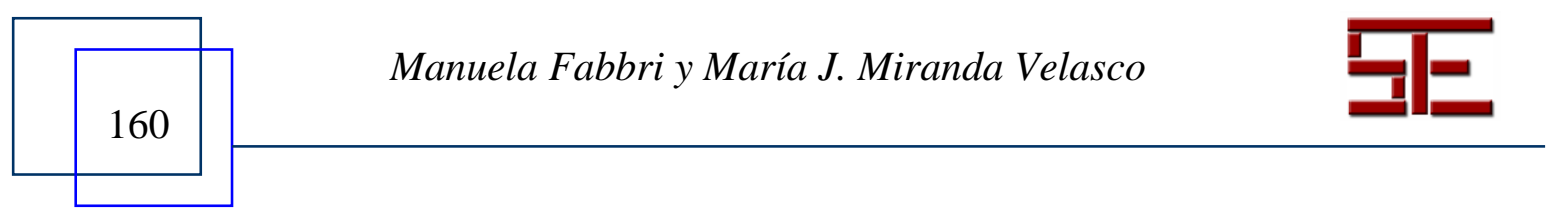




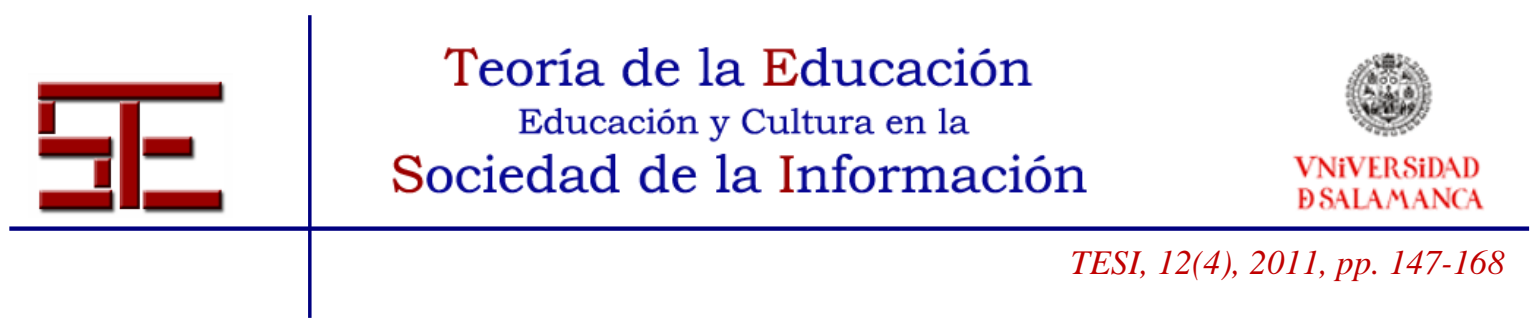

a) Modalidad de apertura del Foro

Como hemos observado, la modalidad de estructura de un Foro en el proceso de enseñanza-aprendizaje influye en la evolución, la participación y, en definitiva, en la calidad del éxito del recurso. Se pueden plantear diversas modalidades de inicio en el Foro en función de la finalidad y de los resultados que se quieren alcanzar. En este sentido, el planteamiento podría ser:

- Muy estructurado. Cuando se pretende controlar un cuadro específico de competencias que han de desarrollar los participantes. En este caso, el Foro debería proponer preguntas estructuradas correspondientes a los saberes objeto de estudio y la descripción detallada de las competencias.

- Medianamente estructurado. Guía y simulación de la discusión por el tutor/docente, basada en procedimientos de aprendizaje relacionados con el constructivismo social. En este caso, es el docente el que tiene que retomar opiniones contrastadas y volverlas a poner en el debate, focalizando los diversos puntos de vista; proporcionar posteriormente materiales y documentación para profundizar en el tema y pedir aclaraciones.

- No estructuradas y provocadoras. Cuando se pretende visualizar la competencia de manera espontánea (las creencias, los sentimientos, etc.). El docente debe evitar intervenir con comentarios que podrían bloquear la espontaneidad de los mensajes producidos por los estudiantes.

b) Inclusión del Foro dentro del programa de la asignatura

En este sentido es importante identificar el Foro como parte de los recursos de aprendizaje facilitados por el docente, y debe ser estructuralmente integrado dentro del plan del curso. Los estudiantes deben tener claro que el trabajo en el Foro también forma parte de sus notas y de su aprendizaje, como también la calidad de su participación en el mismo será objeto de evaluación.

Dependiendo de los objetivos, se puede colocar en diferentes momentos del programa. Por ejemplo, si se coloca al principio como un elemento de provocación, puede servir para identificar los conocimientos previos de los estudiantes.

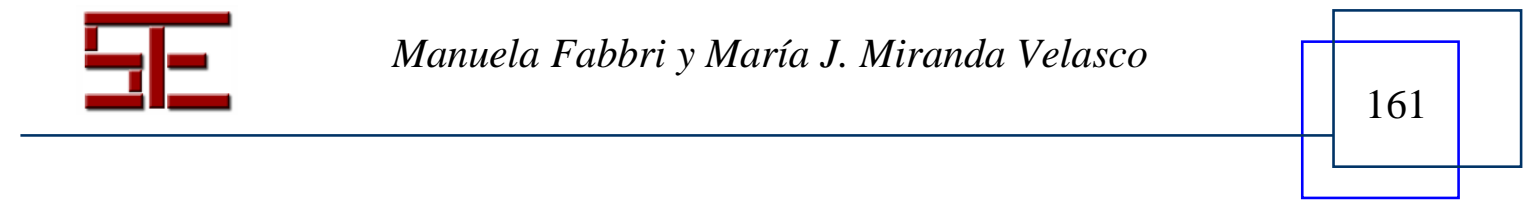




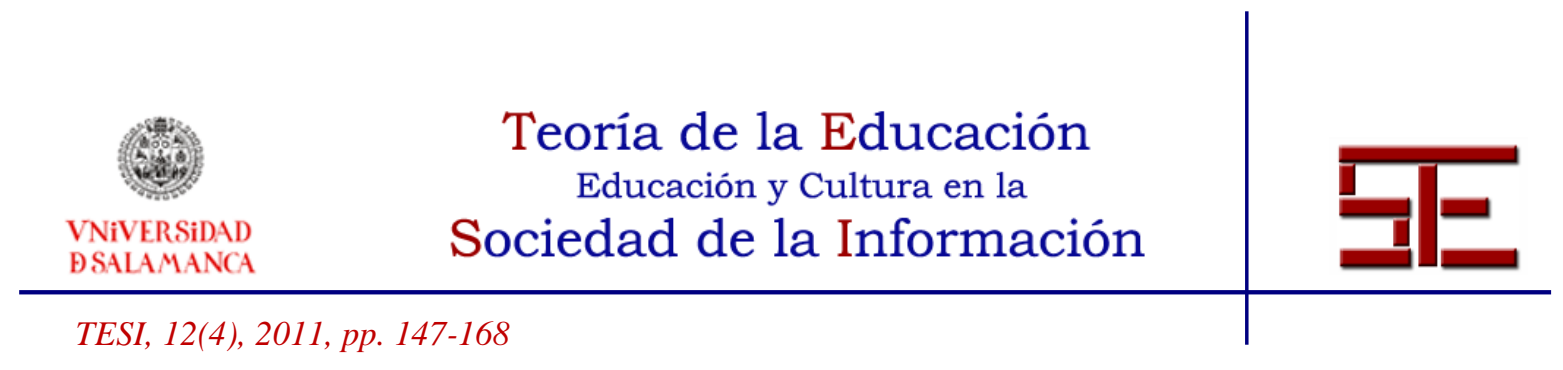

c) Calidad de la comunicación en el foro

Se supone que todos los estudiantes están técnicamente formados para interactuar con la plataforma Moodle. Esta familiaridad con el uso de las tecnologías está ciertamente relacionada con su habituación a la utilización de diferentes recursos de comunicación de carácter tecnológico como son el chat, el e-mail, los SMS, las redes sociales, etc. Sin embargo, la familiaridad para interactuar con las tecnologías parece estar acompañada de una cierta "ligereza" en la expresión escrita. Se encuentran casos que denotan poca atención en cuanto a las reflexiones realizadas. Las argumentaciones son superficiales y no están basadas en una información rigurosa. Además, la ortografía también parece afectada, y se observa una evidente falta de relectura del texto escrito. Aparecen también, en algunos casos, algunas abreviaciones típicas del lenguaje utilizado en los mensajes cortos por móvil.

Por este motivo, algunos profesionales señalan con el dedo contra las nuevas tecnologías, haciéndolas directamente responsables de un "nuevo analfabetismo". Sin en embargo, en la actualidad, se puede comprobar que nunca como ahora los jóvenes han tenido la posibilidad de escribir y de expresarse, para sí mismos, para los demás, y con los demás, por lo que valoramos muy positivamente esta actitud y comportamiento social.

En nuestro trabajo hemos observado que los estudiantes han interactuado entre ellos a través del Foro de una manera básicamente informal; desde un punto de vista emocional y valorativo, expresando sus puntos de vista, sus formas de sentir y a veces también en patente contraste con las orientaciones testimoniales del profesor, despreocupados de los posibles aspectos valorables en sus calificaciones.

d) Potenciación del rol de estudiante

Con el objetivo de implicar y responsabilizar a los alumnos, es necesario monitorizar en alguna medida la marcha de la discusión. Consideramos pertinente intensificar el esfuerzo de flexibilizar el proceso, incentivando momentos de participación activa de los estudiantes, tanto en la fase de iniciación, como en la fase de realización y clausura del mismo Foro. En este sentido, es necesario también prever una intervención final que retome las diversas reflexiones surgidas.

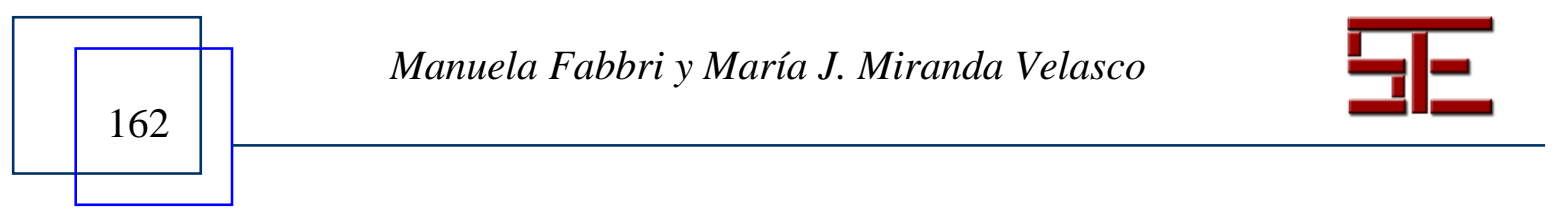




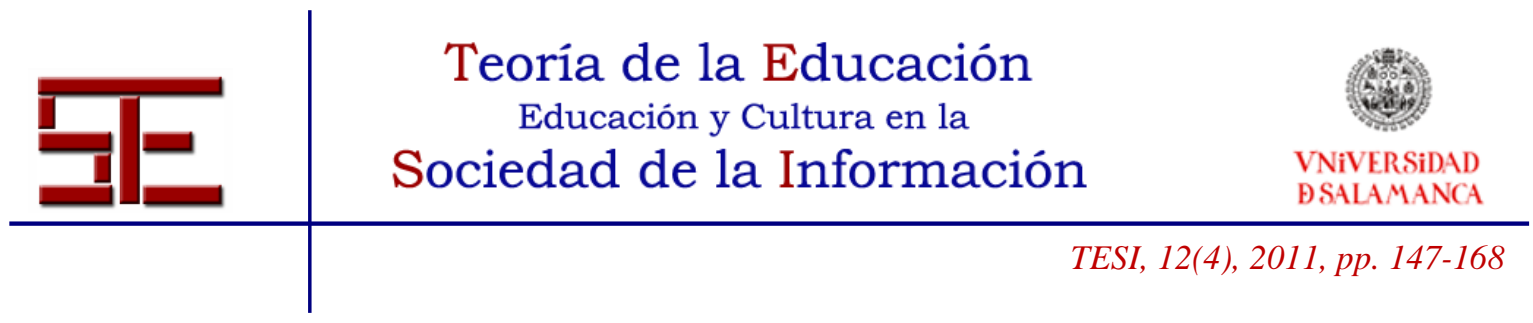

En coherencia con un planteamiento de construcción activa y participativa del conocimiento, cuando un estudiante propone de manera fundamentada un tema interesante, es preciso tenerlo en cuenta y valorarlo para su inclusión en el proceso educativo. Esto puede suponer a su vez un refuerzo y una motivación para aumentar la construcción del conocimiento cooperativamente.

\section{5.- CONCLUSIONES}

A través de la experiencia del Foro descrita podemos concluir que se trata de un recurso de aprendizaje colaborativo con un alto poder educativo y de socialización (Calvani, 2005). Favorece el desarrollo de la capacidad de expresión. Puede ser útil para la adquisición de competencias relacionadas con los valores democráticos y sociales que todo profesional de la educación debe desarrollar (Libro Blanco Comisión Europea, 1995; Bolívar y Guarro, 2007). Finalmente, consideramos que sirve para tomar conciencia de las funciones del Educador Social y Cultural en coherencia con las características y necesidades de vivir en una sociedad global (Estrategia de Lisboa, 2000; Alessandrini, 2001; Castells, 2002; Granieri, 2006).

Como hemos observado en los resultados del segundo año de las intervenciones de los estudiantes, el esfuerzo en el diseño de recursos didácticos adaptados a los recursos tecnológicos concretos, en este caso el Foro, puede mejorar los procesos de enseñanzaaprendizaje.

La inclusión de indicaciones más precisas y exigentes para la realización de los argumentos de los estudiantes está relacionada con la adquisición de competencias de búsqueda de información documental especializada y proporciona mayor calidad al discurso cualitativo producido por el estudiante.

La selección de los dos temas de debate propuestos en la plataforma de la asignatura de Tecnología Educativa, basados en temas de la actualidad cotidiana, cercana a los problemas sociopolíticos actuales, relacionados con los valores educativos que es preciso desarrollar en la formación universitaria, puede ayudar a los estudiantes a tomar una posición activa, contextualizada y comprometida en sus argumentos.

La experimentación de modelos de aprendizaje constructivista ofrece ventajas educativas demostrables relacionadas con las demandas de su rol profesional, al dotarlo

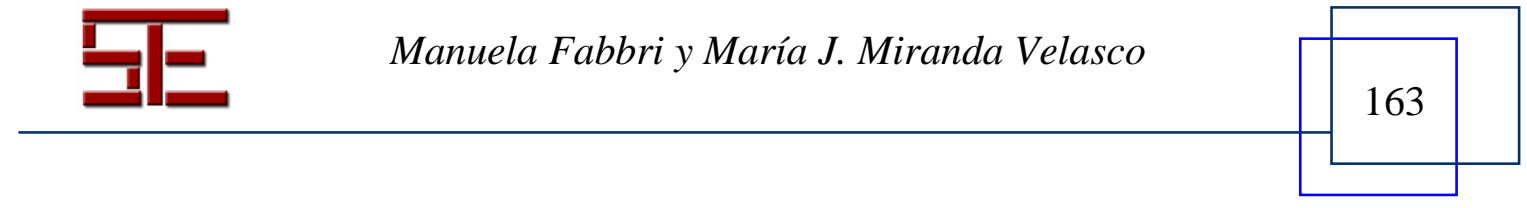




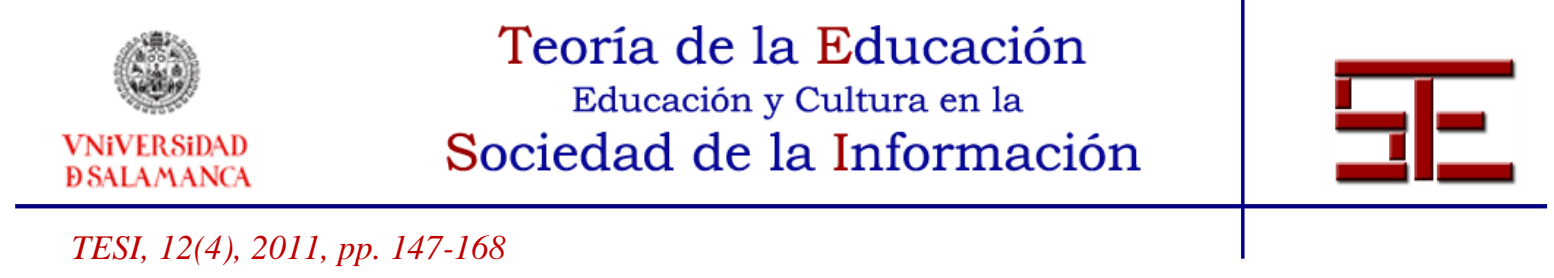

de competencias con las cuales responder de una manera eficaz a los problemas sociales desde una perspectiva educativa.

Otra de las ventajas concretas observadas es que puede ayudar a identificar los posibles prejuicios de los estudiantes relacionados con los prejuicios sociales que no deben reproducir en su intervención educativa profesional. Desde la perspectiva, destacamos la función preventiva y formativa de la universidad. El estudiante será un agente social formado cuyas actitudes y competencias profesionales deben demostrar los valores democráticos que es necesario desarrollar.

Finalmente, llegamos a la conclusión de que a pesar de que el mundo de las redes sociales está comenzando a estar presente en la enseñanza a través de blogs, foros, Facebook, Twitter, a los estudiantes les resulta difícil establecer una cultura de verdaderas "comunidades de aprendizaje" (Brown, Campione, 1994; Flecha y Puigvert, 2007).

\section{BIBLIOGRAFÍA}

Alberici, A. (2002). Imparare sempre nella società della conoscenza. Milano: Bruno Mondadori.

- (2004). Apprendimento di competenze strategiche: l'innovazione dei processi formativi nella società della conoscenza. Milano: Franco Angeli.

Alessandrini, G. (2001). Risorse umane e new economy: formazione e apprendimento nella società della conoscenza. Roma: Carocci.

Berstein, B. (1990). Poder, educación y conciencia. Barcelona: El Roure.

Bertin, G. M. (1968). Educazione alla ragione. Roma: Armando.

Bolívar, A. \& Guarro, A. (Eds.) (2007). Educación y Cultura Democrática: el Proyecto Atlántida (pp. 13-33). Madrid: Wolters Kluwer.

Brown, A. \& Campione, J. (1994). Guided discovery in a community of learners. En: K. McGilly (Coord.). Classroom lessons: Integrating cognitive theory and classroom practice (pp. 229-270). Cambridge, MA: MIT Press.

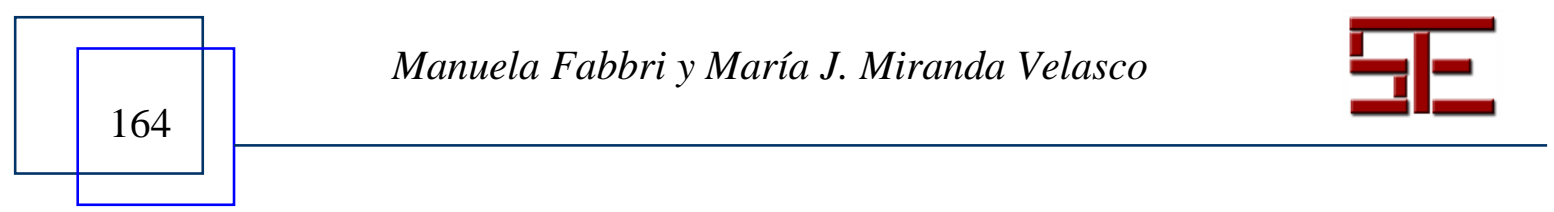




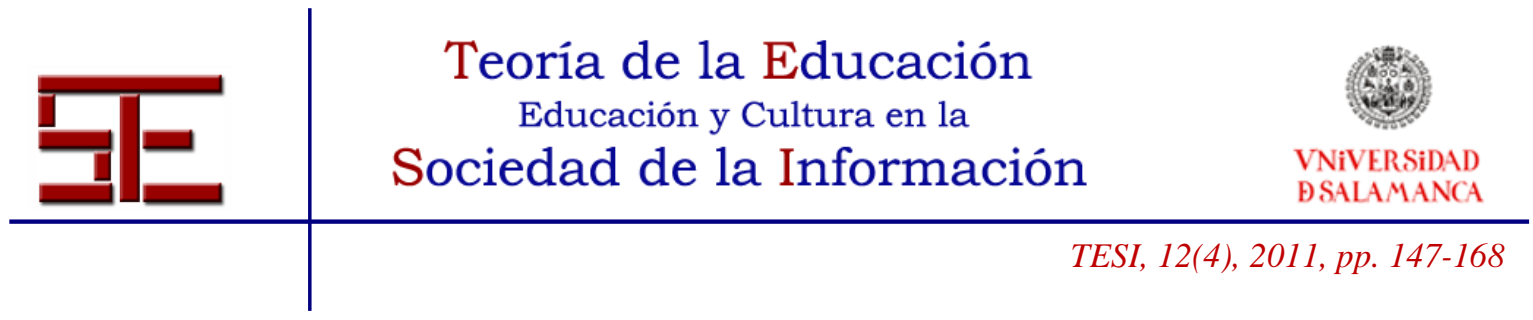

Bruschi, B. \& Ercole, M. (2007). Strategie per l'e-learning. Progettare e valutare la formazione on line. En Calvani, A. (Coord.) Fondamenti di didattica: teoria e prassi dei dispositivi formativi, Roma: Carocci.

Cabero, J. (2005). Reflexiones sobre los nuevos escenarios tecnológicos y los nuevos modelos formación que generan. http://tecnologiaedu.us.es/formaytrabajo/temas/temas.htm.

Calvani, A. (2005). Rete, comunità e conoscenza. Costruire e gestire dinamiche collaborative. Trento: Erickson.

Calvani, A., Sorzio, P. \& Varisco, B. (1997). Uno studio esplorativo di apprendimento cooperativo a distanza [Versión electrónica]. TD - Tecnologie Didattiche, 12, 27-33.

Castells, M. (2002). La nascita della società in rete. Milano: Università Bocconi.

- (2009). Comunicación y Poder. Madrid:Alianza.

Cesareni, D., Ligorio, M. \& Pontecorvo, C. (2001). Discussione e argomentazione in un forum universitario [Versión electrónica]. TD - Tecnologie Didattiche, 3, 55-65.

Colas Bravo, M. P. (1994). La investigación-acción. En Colás, E. y Buendía, L. Investigación Educativa. Sevilla: Alfar.

Commission of the European Communities (2005). Recommendation of the European Parliament and the Council on key Competences for Lifelong Learning. Brussels: CEC.

Costa, M. (2002). Glocal learning. L'economia della formazione. Torino: UTET.

Delors, J. (1996). La educación encierra un tesoro. Informe a la UNESCO de la Comisión Internacional para la Educación para el siglo XXI. UNESCO.

Elliott, J. (1993). El cambio educativo desde la investigación-acción, Madrid: Morata. Fabbri, M. (2009). Insegnare all'Università nella prospettiva del Web 2.0 Il Forum come ambiente di formazione [Versión electrónica]. RPD - Ricerche di Pedagogia e Didattica, 4, 1001-1027.

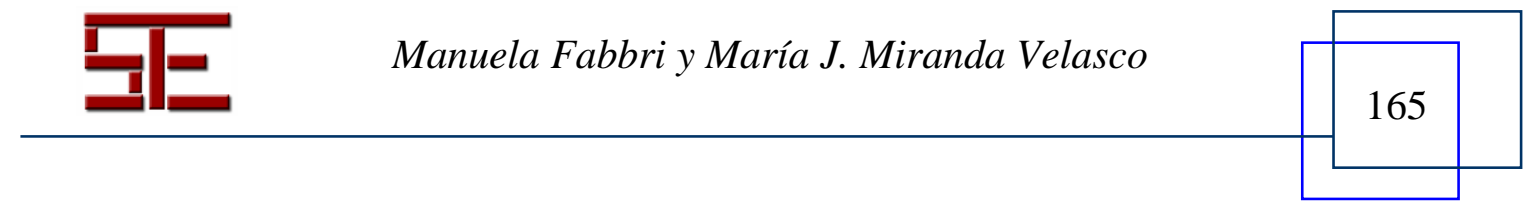




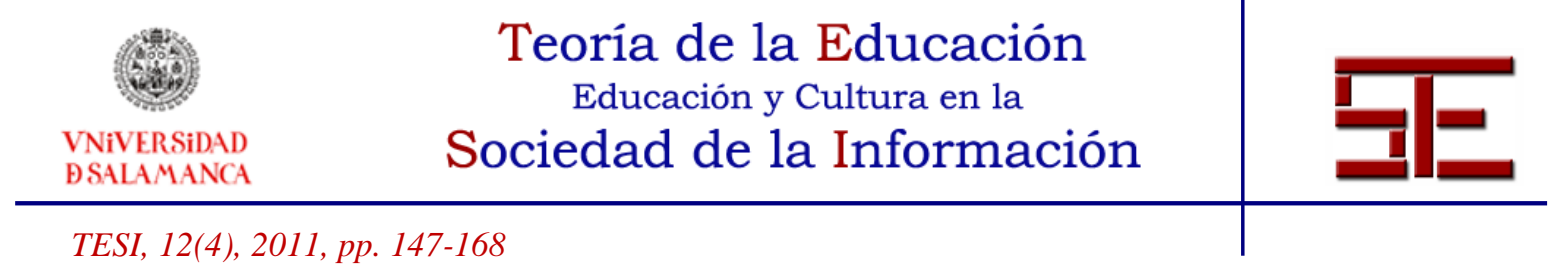

Fabbri, M. Guerra, L. (2010). Las ITC en la Facultad de Ciencias de la Formación de la Universidad de Bolonia: entre Web 1.0 y Web 2.0. En Miranda, M. J., Guerra, L., Fabbri, M. \& López, E. (Coords.) Experiencias universitarias de innovación docente hispano-italianas en el Espacio Europeo de Educación Superior (91-107) Sevilla: Mergablum.

Flecha, R. \& Puigvert, L. (2007). las comunidades de aprendizaje: una apuesta por la igualdad (www.comunidadesdeaprendizaje.net/pdf/flecha_puigvert_02.pdf).

Gattullo, M., Bertolini, P., Canevaro, A., Frabboni, F. \& Telmon V. (1985). Educazione e ragione. Firenze: La Nuova Italia.

Goyette, G. \& Lessard-Hérbert, M. (1988). La investigación-acción. Funciones, fundamentos e instrumentación. Barcelona: Alertes.

Granieri, G. (2006). La società digitale. Bari: Laterza.

Guerra L. (Coord.) (2010). Tecnologie dell'educazione e innovazione didattica. Azzano San Paolo (BG): Edizioni Junior.

- (2011). Formazione e social networking. FOR, 85, 8-10.

Habermas, J. (1972). La ciencia y la tecnología como ideología. Madrid: Alianza Editorial.

Lewin, K. (1973). Action research and minority problems. En K. Lewin, Resolving Social Coflicts: Selected Papers on Group Dynamics. London: Souvenir Press.

Libro Bianco della Commissione Europea (1995). Insegnare e apprendere: verso la società conoscitiva. Édith Cresson.

Nichols, R. \& Allen-Brown, V. (1996) Critical theory and education technology. In D. Jonassen (ed.), Handbook of research for Educational Communications and Technology. New York: Mac-Millan.

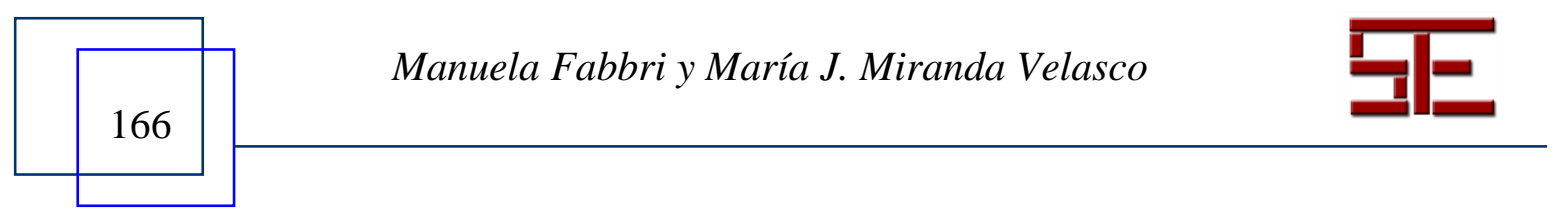




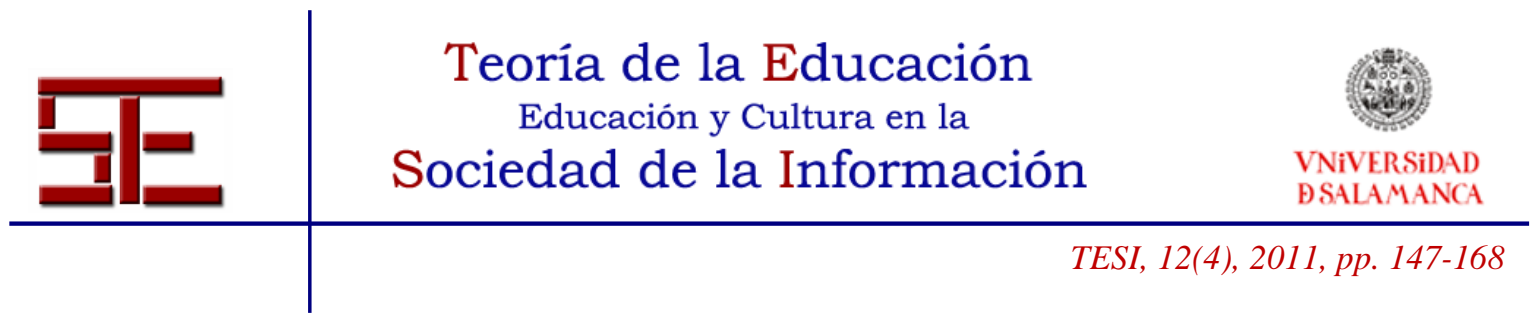

Maeran, G. (2011). Società della conoscenza e social-networking: un'esperienza di forum. Memoria para optar al título de Doctor, Pedagogia, ciclo XXII, Alma Mater Studiorum, Universitat de Bolonia, Bolonia, Italia.

McLuhan, M. \& Powers, B. (1988). The Global Village: Transformations in World Life and Media in the 21st Century. Oxford: University Press.

Miranda, M. J. \& López, E. (2006). Las nuevas tecnologías de la información y la comunicación y la Educación Superior: nuevos desafíos, nuevas posibilidades para el desarrollo de la ciudadanía. Revista Educação, 12, 61-79.

Miranda, M.J. (2010). El desarrollo de la Universidad en el Espacio Europeo. En: Miranda, M. J., Guerra, L., Fabbri, M. \& López Meneses, E. (coords.), Experiencias universitarias de innovacion docente hispano-italianas en el espacio europeo de educacion superior (pp. 11-41). Sevilla: Mergablum.

Miranda, M. J., Fabbri, M. \& Guerra, L. (2010). Recursos tecnológicos y ensenanzaaprendizaje de competencias en educación social. Una experiencia internacional de constructivismo social en el EEES. Educación Social, 44, 55-73.

Miranda, M. J., Guerra, L., Fabbri, M., Moríñigo \& López „E. (2010). Experiencias de innovación docente sobre interculturalidad "Acoglienza e integrazione". En Miranda, M. J., Guerra, L., Fabbri, M. \& López Meneses, E. (Coords.). Experiencias universitarias de innovación docente hispano-italianas en el Espacio Europeo de Educación Superior (pp.165-176). Sevilla: Mergablum.

OECD/SFSO/DeSeCo. (2002). Definition and Selection of Competencies:Theoretical and Conceptual Foundations (DeSeCo). Annual Report2001. http://www.deseco.admin.ch/bfs/deseco/en/index/01.parsys.70925.downloadList.59988. DownloadFile.tmp/2001 annualreport.pdf.

Pavan, A. (2008). Nelle società della conoscenza. Il progetto politico dell'apprendimento continuo. Roma: Armando Editore.

Pèrez A. y Mugny, G. (1993). Influences sociales. La théorie de l'elaboration du conflict. Laussanne: Delachau et Niestlè.

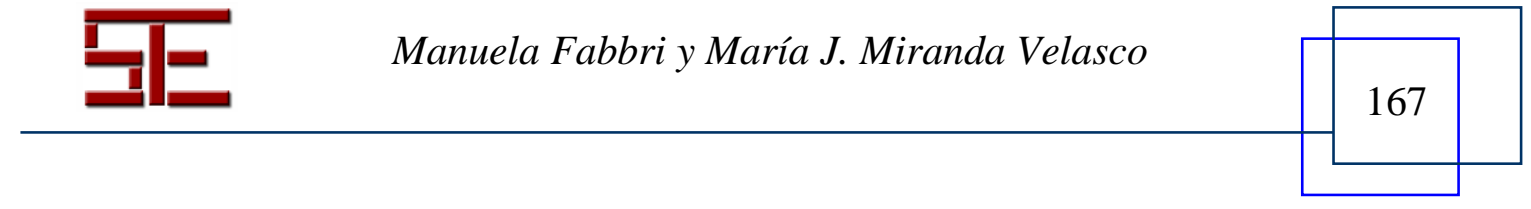




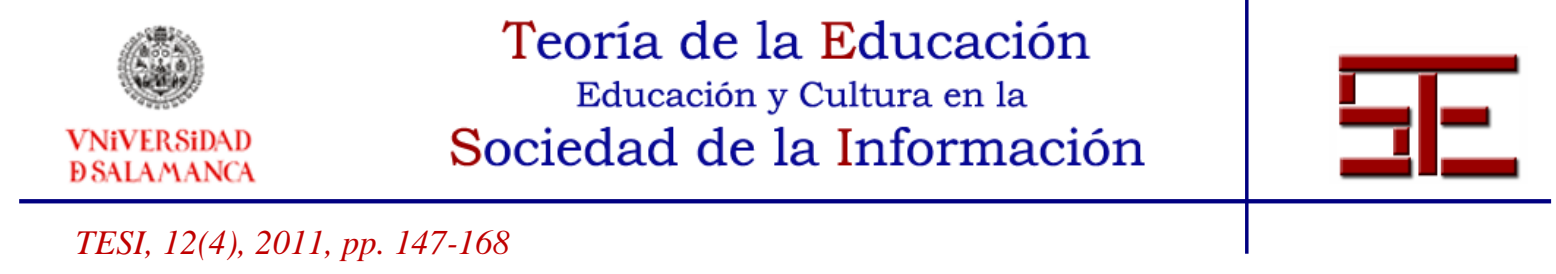

Seoane, A., García Carrasco, J. \& García Peñalvo, F. (2007). La tutoría online como elemento estratégico para una e-formación de calidad. Revista Electrónica Teoría de la Educación. Educación y Cultura en la Sociedad de la Información, 5-8. Monográfico 2007. Vol. Extraordinario.

UNESCO (2008). Estándares de Competencias en TIC para docentes. Disponible en: http://www.eduteka.org/EstandaresDocentesUnesco.php. (Consultado: 10-11-2009).

Valverde, J. (2010). El tutor on-line: funciones, roles y tareas. En: En Miranda, M. J.; Guerra, L.; Fabbri, M. \& López Meneses, E. (Coords.) (2010). Experiencias universitarias de innovación docente hispano-italianas en el Espacio Europeo de Educación Superior (pp.43-73). Sevilla: Mergablum.

\footnotetext{
${ }^{1}$ La laurea de Educatore Sociale (3 años) e Culturale es equivalente en España al título de Diplomatura en Educador Social.

${ }^{2}$ La Escuela del problematicismo Pedagogico Bolognese fue fundada por Giovanni Maria Bertin, primer director del actual Instituto de Ciencias de la Educación de la Universidad de Bolonia. Su obra fundamental se recoge en: Bertin, G. M. (1968). Educazione alla ragione. Roma: Armando. El pensamiento de Bertin se sigue desarrollando en profundidad por colegas y colaboradores actuales tal como se refleja la amplia producción científica de los diferentes autores: Gattullo, M., Bertolini, P., Canevaro, A., Frabboni, F. y Telmon V. (1985). Educazione e ragione, Firenze: La Nuova Italia.

${ }^{3}$ Para poder profundizar y ver el curso en su totalidad: cfr: http://www.moodle.unibo.it/, alla sezione Facoltà di Scienze della Formazione/Lauree Triennali/ tecnologie dell'educazione.

4 Esta ampliación se hizo a través de una ampliación de la investigación de parte del grupo de investigación de la Universidad de Bolonia, compuesto por Luigi Guerra, Manuela Fabbri y Gilda Maeran, basándose en el importante trabajo de investigación previamente realizado por Cesareni, Ligorio y Pontecorvo, 2001.
}

Para citar el presente artículo puede utilizar la siguiente referencia:

Fabbri, M. y Miranda, M. J. (2011). Formación y educación cultural en la sociedad del conocimiento. Análisis evolutivo de una estrategia didáctica colaborativa en educación superior. Revista Teoría de la Educación: Educación y Cultura en la Sociedad de la Información. 12(4), 147-168 [Fecha de consulta: dd/mm/aaaa].

http://campus.usal.es/ revistas_trabajo/index.php/revistatesi/article/view/8531/8617

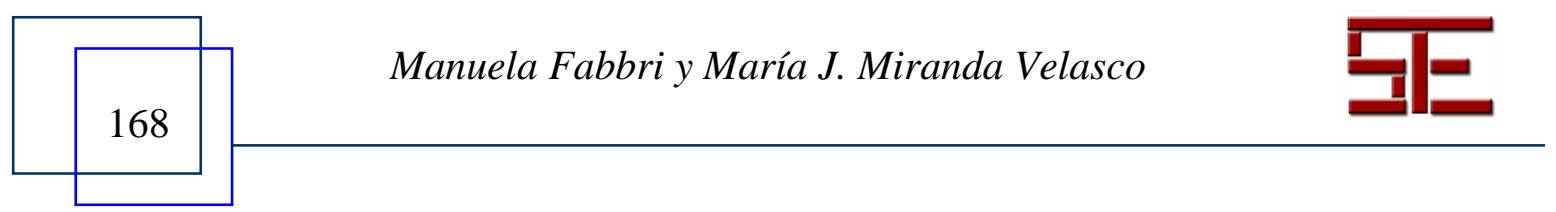

